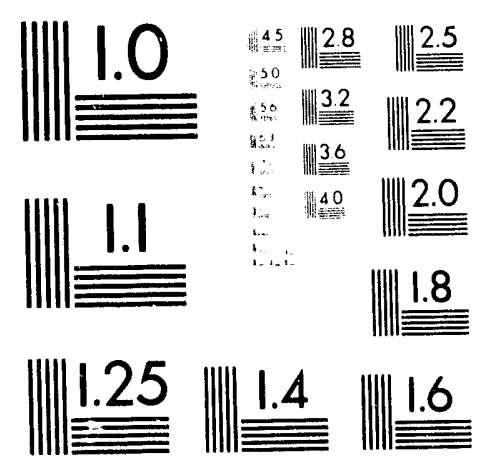



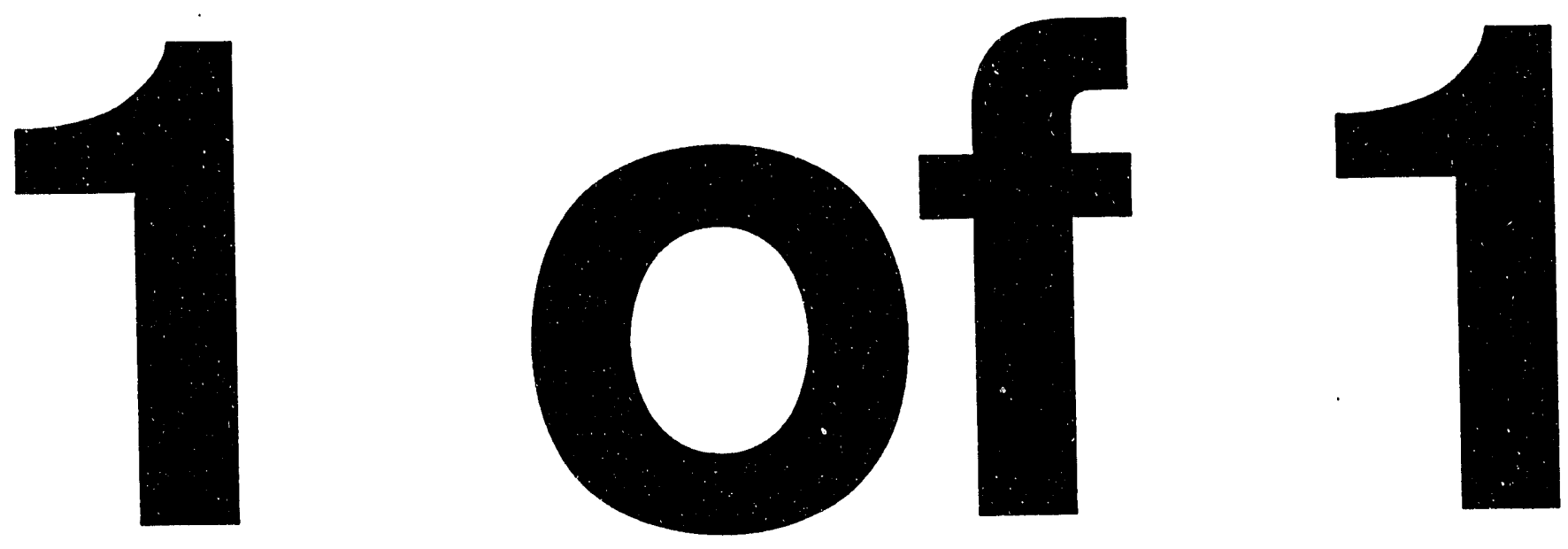
1993 PART ICLE ACCELERATOR CONF

WASHINGTON, DC

MAY $17.20,1993$

\title{
RHIC Beam Position Monitor Assemblies *
}

\author{
P.R. Cameron, M.C. Grau, W.A. Ryan, T. J. Shea, and R.E. Sikora \\ Brookhaven National Laboratory \\ Upton, NY 11973 USA
}

Abstract

Design calculations, design details, and fabrication techniques for the RHIC BPM Assemblies are discussed. The 69 $\mathrm{mm}$ aperture single plane detectors are $23 \mathrm{~cm}$ long short-circuited $50 \mathrm{ohm}$ strip transmission lines subtending 80 degrees. They are mounted on the sextupole end of the Corrector-Quadrupole-Sextupole package and operate at liquid helium temperature. The $69 \mathrm{~cm}$ aperture was selected to be the same as that of the beampipe in the CQS package, the $23 \mathrm{~cm}$ length is a compromise between mechanical stability and electrical sensitivity to the long low-intensity.proton and heavy ion bunches to be found in RHIC during commissioning, and the 80 degree subtended angle maximizes linear aperture. The striplines are aligned after brazing to maintain electrical-to-mechanical centers within $0.1 \mathrm{~mm}$ radius, eliminating the need for individual calibration. Because the cryogenic feedthrus isolate the UHV beam vacuum only from the HV insulating vacuum, and do not see liquid helium, a replaceable mini-Conflat design was chosen to simplify fabrication, calibration, and maintenance.

\section{Performance Requirements}

The RHIC Design Manual specifies that the beam position measurement be accurate within 130 microns from the center of the sextupole field to the number disp'ayed in the control room (because of the strong chromaticity correction in RHIC the most stringent position requirement for the BPM is relative to the sextupole). There are three main sources of error in the position measurement. First are errors from the center of the sextupole field to the position of the dowel holes in the plate upon which the BPM is mounted. Second are errors in the location of the BPM mechanical center (determined by the dowel pins) relative to the BPM electrical cen$\operatorname{ter}^{1}$ (determined by the geometry of the striplines). And finally, there are errors in the electronics ${ }^{2}$. These errors add in quadrature, which suggests that error from each source should be less than about 100 microns. This accuracy in the position of the BPM electrical center relative to the mechanical center is easily accomplished using the bumping alignment technique described in Section III.

\section{Design Details and Fabrication Techniques}

A typical RHIC Beam Position Monitor is shown in Figure 1. The non-directional shorted stripline design minimizes vacuum penetrations, and adapts well to economical fabrication and alignment techniques.

- Work supported by the U.S. Department of Energy

\section{A. Brazement}

The brazement is constructed of type $316 \mathrm{~L}$ stainless stcel, which is copper brazed in a hydrogen furnace. Because the brazing temperature is above the annealing temperature for $316 \mathrm{~L}$, the mini-Conflat flanges, the buffer volume bellows, and the tailpiece bellows are welded on after brazing. The mini-ConFlat flanges are TIG welded. The buffer volume and tailpiece bellows are EB welded to minimize thermal distortion.

The flange is machined from a $316 \mathrm{~L}$ forging to eliminate the possibility of piping from the liquid helium buffer volume to the beam vacuum. Two alignment pins are pressed into the flange, and one of the two pins is a diamond pin to eliminate over-constraint.

The striplines are wire EDM cut from seamless 316 tube redrawn to size inside and out. The redrawing operation delivers a tube accurate enough to require machining on the circumference only at the braze joint, where tolerances are closely controlled to maintain between 13 and 38 microns radial clearance. A groove is machined in the stripline adjacent to the braze joint to concentrate stresses and localize yielding during the alignment bumping procedure. ANSYS 3D plastic analysis of this region is in progress. Stripline impedance was calculated to be 50 ohms using PE2D, ANSYS, HFSS, and EMAS.

The housing is progressively drawn to shape from $316 \mathrm{~L}$ welded tubing. Again, the drawing operation delivers a part accurate enough to require circumferential machining cnly at the braze joint. The material thickness was selected to satisfy the ASME Boiler Code.

The buffer volume bellows mechanically isolates the BPM from displacements of the buffer volume during the 1.9 MPascal (275 psi) quench. Performance of this bellows during quench was analyzed using a program written ${ }^{3}$ specifically for bellows analysis. Several different geometries were ANSYS analyzed in this region before settling on the bellows.

Despite precise fixturing and good attention to welding technique, it has not been possible to maintain satisfactory aligniment when accomplishing the BPM-to-beampipe weld. The beampipe has a $3 \mathrm{~m}$ long lever arm on the BPM. The convolutions in the tailpiece region provide a hinge which prevents the generation of excessive stresses and the resulting distortion. The annular space between the two bellows is ported to the insulating vacuum. The outer bellows is required by a RHIC design specification that insulating vacuum be interposed between beam vacuum and liquid helium at all welds. Both bellows are designed ${ }^{3}$ to resist quench pressure.

$$
\left\{\begin{array}{l}
n \\
3
\end{array}\right.
$$$$
\text { DISTRIBUTION OF THIS DQCUUMENT IS UNLIMITED }
$$ 




Figure 1. Typical BPM showing surrounding Buffer Volume and adjacent CQS Assembly

\section{B. Contacts}

The contact and feedthru geometry is shown in Figure 2. The bellows spring portion of the contact is gold plated electrodeposited sulfur free nickel, which is EB or laser welded to the stainless steel post. The spring rate of the bellows is 12.5 $\mathrm{lb} / \mathrm{in}$, the maximum compression is $1.8 \mathrm{~mm}$, the working compression is $1 \mathrm{~mm}$, and the contact force is $2.5 \mathrm{~N}$ (9 ounces). The maximum operating temperature is limited to $180 \mathrm{C}$ by annealing of the nickel. While the impedance match to the feedthru is the responsibility of the manufacturer, some modelling was done using HFSS.

\section{Feedthrus}

The feedthru is incorporated into a mini-ConFlat package. Two different feedthru designs are being evaluated. The more conservative conventional design uses ceramic dielectric metallized and brazed to a thin kovar strain relief which is welded to the stainless steel flange. This design is relatively complicated and expensive, but is a proven performer. The newer design uses lithium silicate glass dielectric which is doped to match the expansion coefficient to stainless steel, and a glass-to-metal seal. This design is more simple and economical and has better RF properties, but has not yet been used in a major accelerator.

A total of 50 prototype feedthrus were purchased from four vendors, equally split between ceramic and glass dielectric. VSWR and S parameters were measured, in good agreement with HFSS calculations. Based upon these measurements and calculations, the specification for the production feedthru requires VSWR of less than 1.1 from DC through $3 \mathrm{GHz}$. Reliability of the SMA connector was poor in some of the feedthrus, in some instances because the contact spring material appeared to have been annealed during manufacture, and in others because of poor contact geometry. Reliability of this connector will receive close attention during acceptance testing of the production feedthrus.

All of the prototype foedthrus were cryogenically tested by direct immersion in liquid nitrogen, followed by warming to room temperature in ambient air. Leak tightness was monitored throughout the cycle, and this cycle was repeated five times. No failures of the dielectric-to-metal bond were observed with either glass or ceramic. This thermal cycling will be part of the acceptance testing for all production feedthrus.

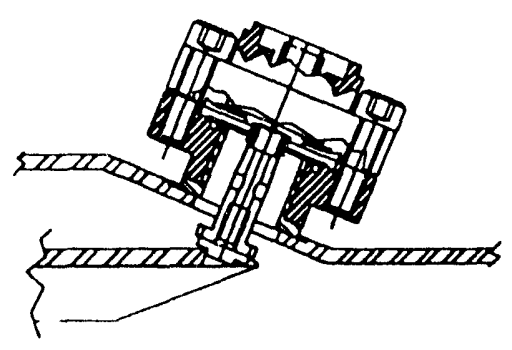

Figure 2. Contact and Feedthru Geometry 


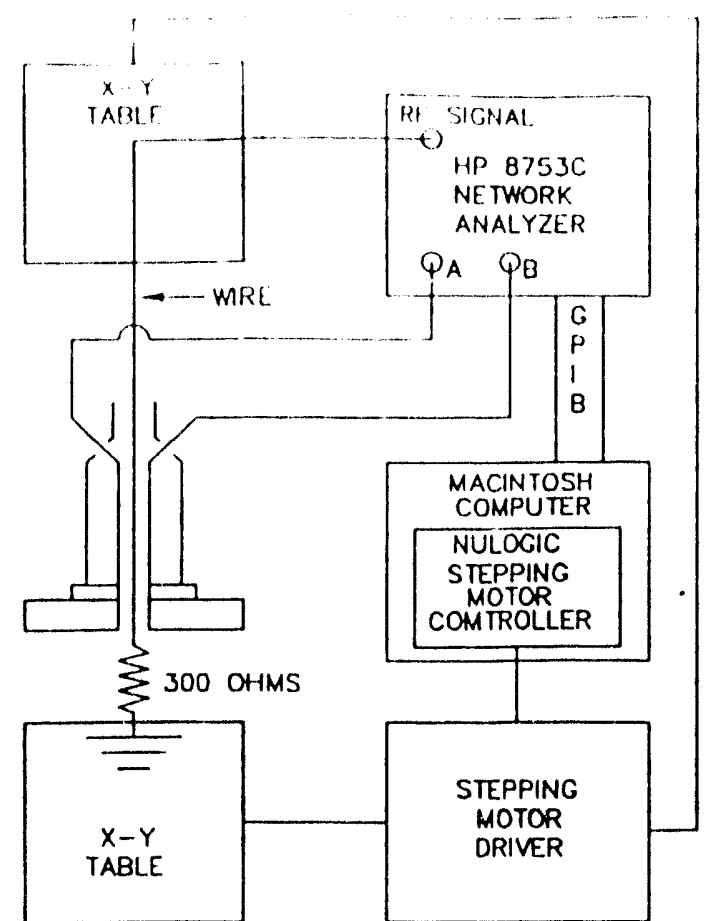

Figure 3. Block Diagram of the Mapping System

\section{Cables}

The cables must be radiation resistant, mechanically flexible, cryogenically compatible, have low heat leak and VSWR, and most important, have good electrical stability. In addition to minimal as-manufactured variations, the cable must be electrically stable when subjected to the above mentioned radiation, flexing, and temperature gradients. Dielectric materials under consideration include silicon dioxide, magnesium oxide, ultem (polyimide), siltem, and tefzel. Silicon dioxide cables have been tested, and seem satisfactory. An experiment $t^{4}$ to measure the radiation damage characteristics of PEEK at liquid helium temperatures resulted in a violent explosion after a dose of about 0.5 gigarad. While this dose is much higher than any expected in accelerator operations, we are not presently actively pursuing this material.

\section{Testing of the Assembly}

\section{A. Calibration of the BPM}

A block diagram of ine mapping system is shown in Figure 3. LabVIEW software running on the Macintosh computer controls the NuLogic stepping motor controller and the network analyzer. The position of the signal wire is initially established with a precision fixture aligned by the dowel holes on the mounting plate. The positions of the wire when it makes electrical contact with the fixture alignment points are recorded, then the fixture is rotated 180 degrees and the results of the two measurements are averaged. Using this method it is possible to position the wire with an accuracy of about 25 microns.
The alignment fixture is then replaced by the BPM, and the pesition of the electrical center of the BPM is measured relative to the mechanical center. The measured transfer function is about $0.7 \mathrm{~dB} / \mathrm{mm}$. The appropriate feedthru is then removed and replaced with an assembly consisting of a micrometer welded into a mini-Conflat llange. The micrometer is cranked in to yield the stripline. Yielding begins at about $2 \mathrm{~mm}$ deflection. After bumping the feedthru is replaced and the position of the electrical center is remeasured. If necessary, the bumping procedure is repeated. Using this method the required $0.1 \mathrm{~mm}$ position tolerance is easily attained.

\section{B. Thermocycling}

Using a test setup similar to that shown in Figure 3, a prototype BPM was thermally cycled from room temperature to liquid nitrogen temperature and back to room temperature. Measurement of the impedance of the striplines indicates that movement of the striplines relative to the housing was less than 25 microns. This measurement will be repeated with a production BPM both at liquid nitrogen and at liquid helium temperatures.

\section{Conclusions}

A simple, robush, accurate, and economical design for a cryogenic BPM has been developed. The possibility of making yet further gains in accuracy with improved survey techniques is under active investigation.

\section{References}

[1] The possibility of greatly reducing the first two types of errors by surveying the BPM electrical center directly to the sextupole magnetic center is discussed in:

M. Goldman, R. Sikora, and T. Shea, "Preliminary Studies on a Magneto-Optical Procedure for Aligning RHIC Magnets", these proceedings.

[2] W. Ryan, T. Shea, P. Cerniglia, and C. Degen, “A Prototype BPM Electronics Module for RHIC", these proceedings.

[3] C. M. Lac, private communication.

[4] Bom Soon Lee, private communication.

\section{DISCLAIMER}

This report was prepared as an account of work sponsored by an agency of the United States Government. Neither the United States Government nor any agency thereof, nor any of their employees, makes any warranty, express or implied, or assumes any legal liability or responsibility for the accuracy, completeriess, or usefulness of an information, apparalus, product, or process disclosed, or represents that its use would not infringe privately owned rights. Reference herein to any specific commercial product, process, or service by Irade name, trademark, manufacturer, or otherwise does not necessarily constitute or imply its endorsement, recommendation, or favoring by the United States Government or any agency thereof. The views and opinions of authors expressed herein do not necessarily state or reflect those of the United States (jovernment or any agency thereof. 



$11 / 10 / 93$
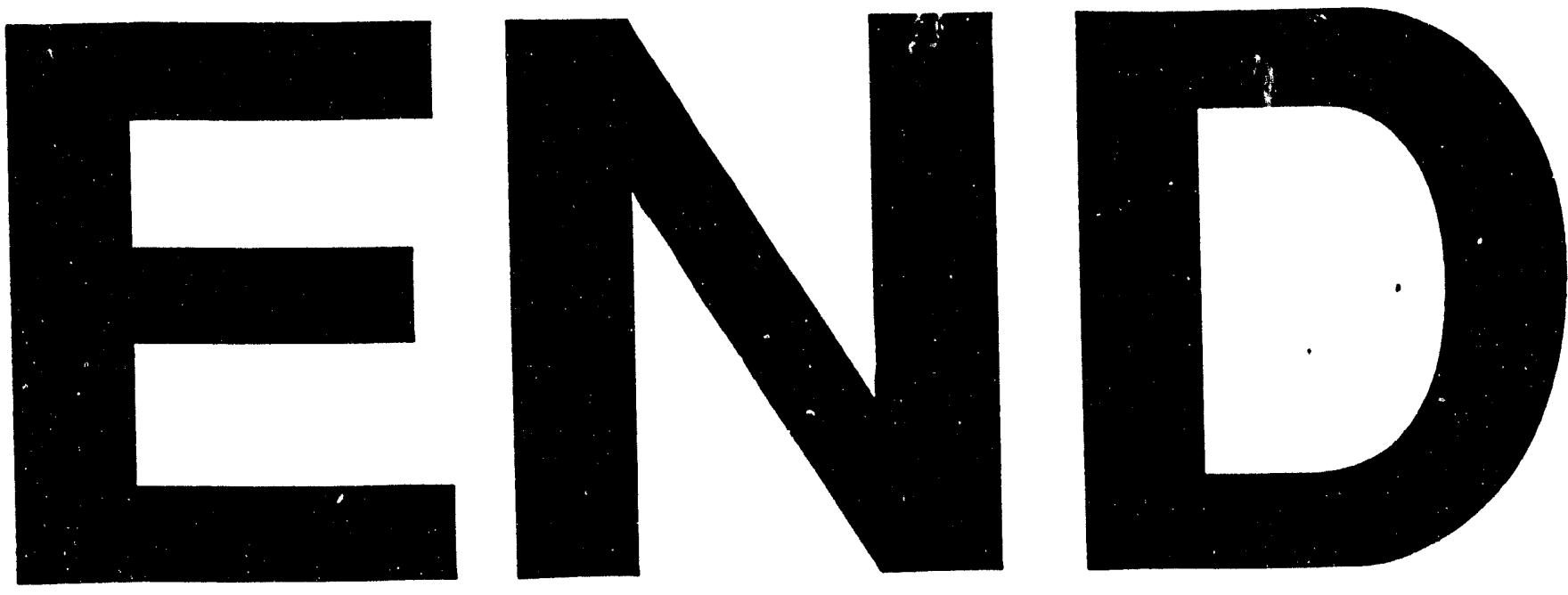


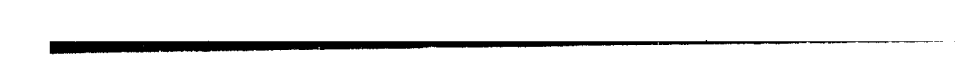

